\title{
Histopathological Study of Gynecological Cancers in Patients Admitted to Baqiyatallah Hospital in Tehran during 2011-2019
}

\author{
Seyedeh Razieh Hashemi ${ }^{1,5}$ (D) , Mohammad Reza Akbari² ${ }^{2}$ Zahra Soleimani ${ }^{1,3,4, *}$ (iD) \\ ${ }^{1}$ Assistant Professor, Department of Obstetrics and Gynecology, Baqiyatallah University of Medical Sciences, Tehran, Iran \\ ${ }^{2}$ Medical Student, Student Research Committee, Baqiyatallah University of Medical Sciences, Tehran, Iran \\ ${ }^{3}$ Nephrology and Urology Research Center, Baqiyatallah University of Medical Sciences, Tehran, Iran \\ ${ }^{4}$ Fetal Health Research Center, Hope Generation Foundation, Baqiyatallah University of Medical Sciences, Tehran, Iran \\ 5 Trauma Research Center, Baqiyatallah University of Medical Sciences, Tehran, Iran
}

* Corresponding Author: Zahra Soleimani, Department of Obstetrics and Gynecology, Baqiyatallah University of Medical Sciences, Tehran, Iran. Email: z.soleimanii2018@gmail.com

Received: 19.12 .2020

Accepted: 13.04.2021

\section{How to Cite this Article:}

Hashemi SR, Akbari MR,

Soleimani Z. Histopathological

Study of Gynecological Cancers in Patients Admitted to Baqiyatallah Hospital in Tehran during 2011-2019. Avicenna J Clin Med. 2021; 28(1): 42-48. DOI: $10.52547 /$ ajcm.28.1.42

\section{Abstract}

Background and Objective: Each type of cancer has its own histopathological findings that determine the type of treatment regimen and the course of the disease. The present study aimed to histopathologically determine the types of gynecological cancers in patients admitted to Baqiyatallah Hospital over a nine-year period.

Materials and Methods: This retrospective study was conducted based on hospital data of patients who were admitted to Baqiyatallah Hospital and underwent any procedure, treatment, and diagnosis of gynecologic cancers during 2011-2019. Patients' information was extracted from hospital integrated archiving system.

Results: A total of 404 patients were diagnosed with gynecological cancers between 2011 and 2019. Among these cancers, ovarian cancer with 212 (52.5\%) patients was the most common gynecological cancer. Among patients with ovarian cancer, Serous Adenocarcinoma and Endometrioid Adenocarcinoma with $137(64.6 \%)$ and $29(13.7 \%)$ cases had the highest frequency of pathology, respectively. The mean (standard deviation) age of the patients with gynecological cancer was 55.88 (11.51) years. During the study period, there was a relatively increasing trend for ovarian cancer during 2011-19.

Conclusion: ovarian cancer with Serous Adenocarcinoma was the most common cancer diagnosed in patients admitted to Baqiyatallah Hospital during 2011-19. The relatively increasing trend of this type of female genital cancers during this 9-year period and the observed age pattern highlight the importance of implementing timely screening and treatment programs.

Keywords: Gynecological Neoplasms, Histopathology, Ovarian Neoplasms 
dol: $10.52547 / \mathrm{ajcm} \cdot 28.1 .42$

\title{
بررسى هيستوياتولوزى سرطان هاى زنيكولوزيك در بيماران بسترى شده در بيمار ستان

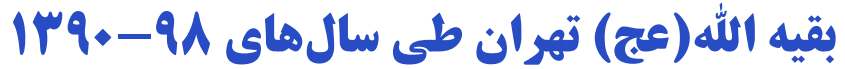

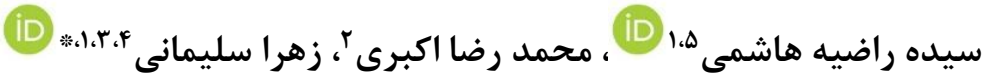

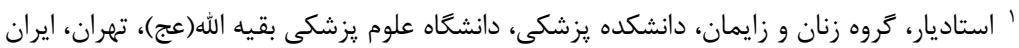

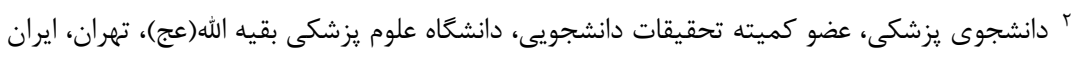

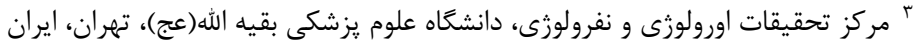

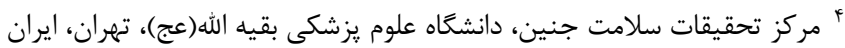

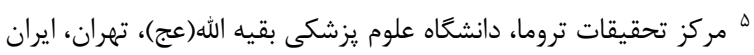
* نويسنده مسئول: زهرا سليمانى، كروه زنان و زايمان، دانشكده يزشكى، دانشكاه علوم يزشكى بقيه الله(عج)، تهران، ايران. ايميل: z.soleimanii2018@gmail.com

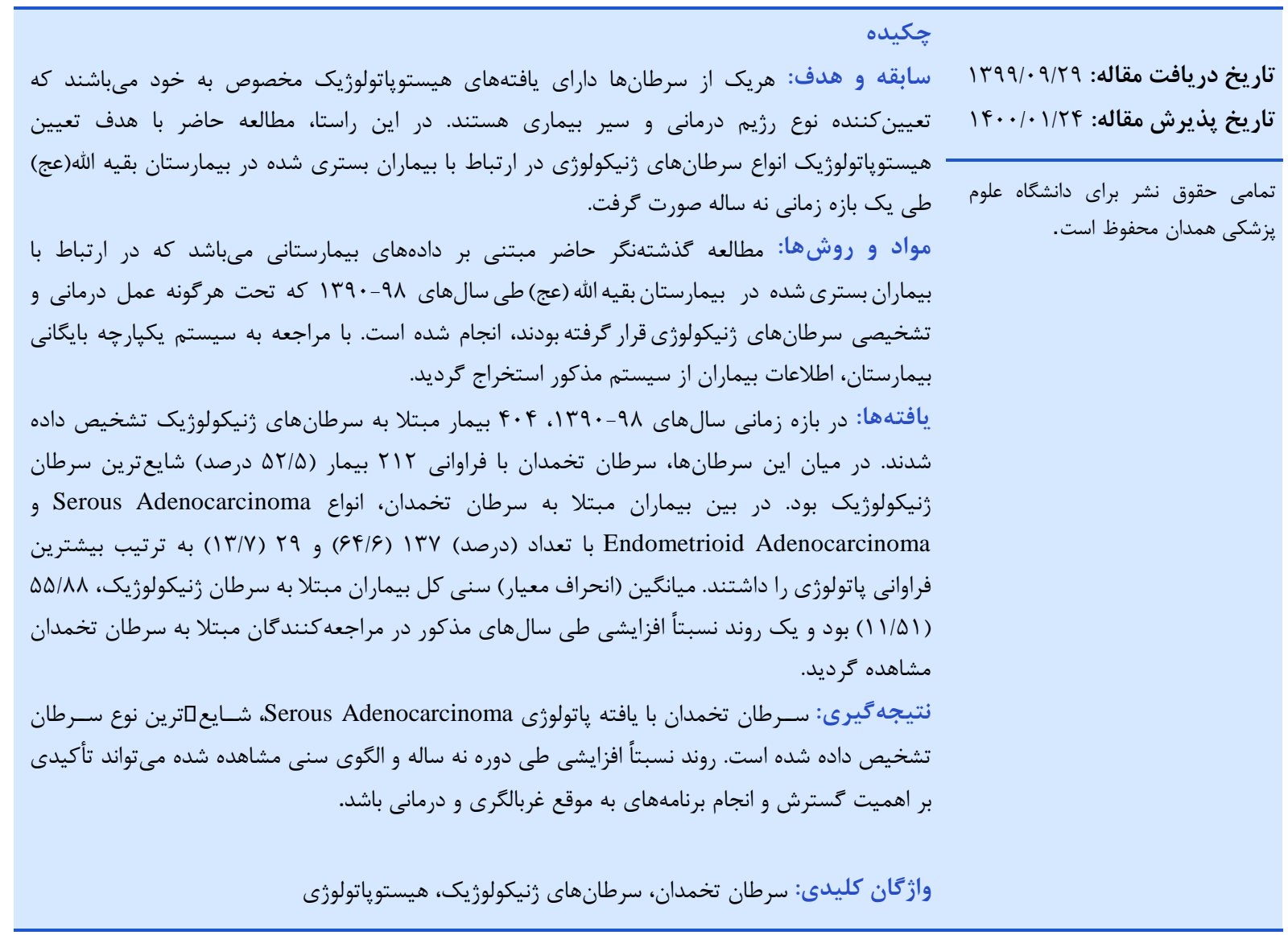

سبك زندكى غربى و افزايش در صد جمعيت سالمندى منجر به

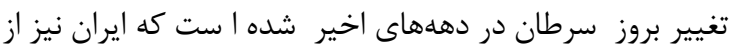

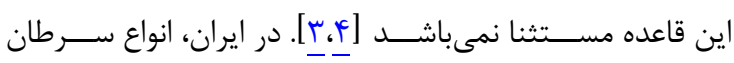

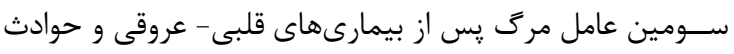
ترافيكى است [ـ-_ه]].
سرطان يك مع ضل عمده سلامتى در ك شورهاى در حال

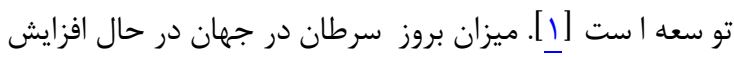

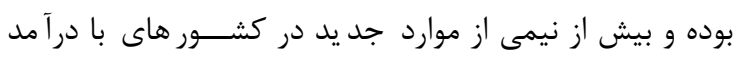

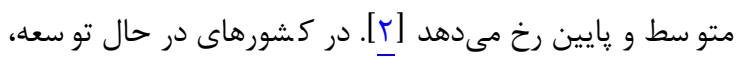

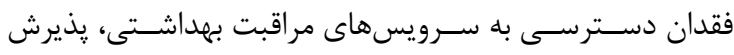


بيمار انى كه به صورت سريايى مر اجعه كرده و بسترى نشده بودند،

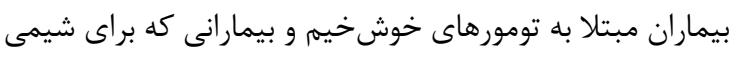

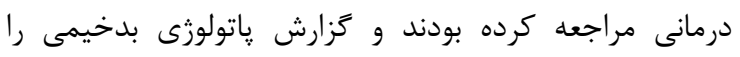

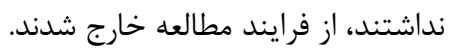

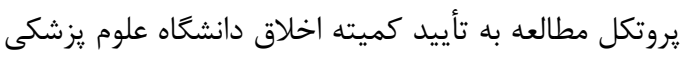

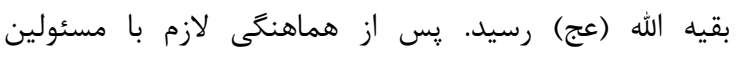

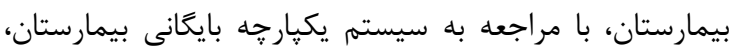

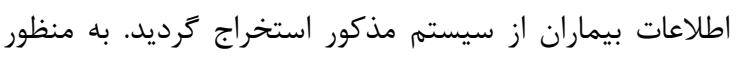

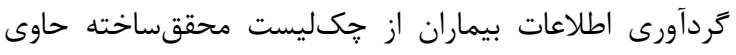

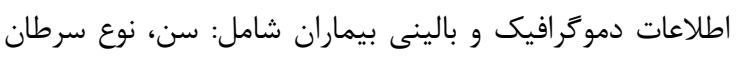

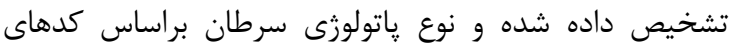

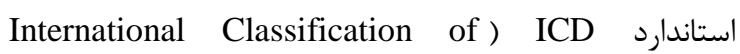
(Diseases ) (ستفاده كرديد. براى توصيف متغيرها از ميانكَين (انحراف معيار) و و تعداد

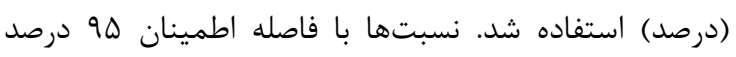

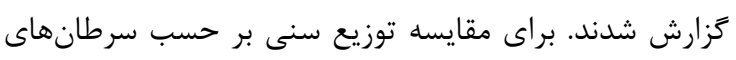

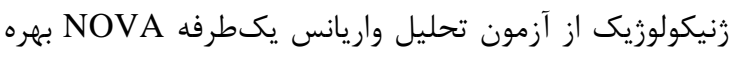

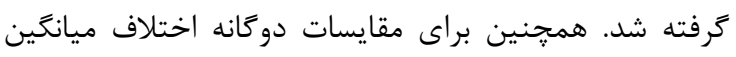
سنى در سرطانهاى رنيكولوزيك از آزمون تعقيبى (Post hoc) از نوع توكى (Tukey) استفاده كرديد. سطح معنادارى آزمونها

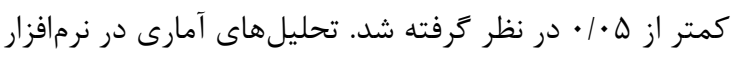
S S S DSS 16

\section{يافته ها}

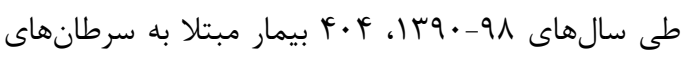

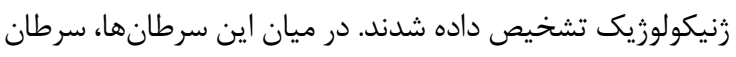

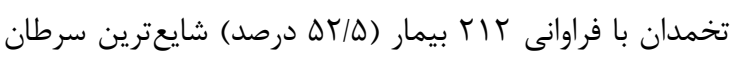

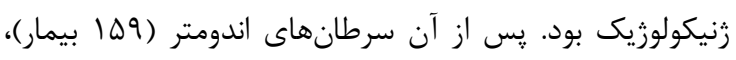

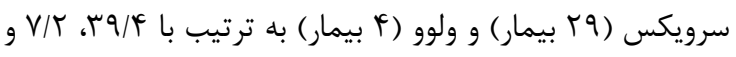

$$
\text { ا درصد داراى بيشترين فراوانى بودند. }
$$

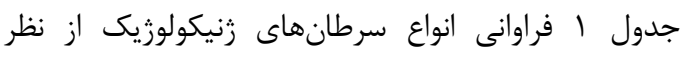

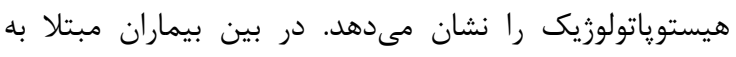

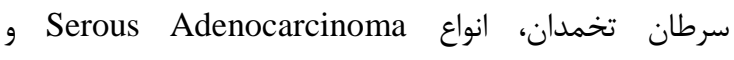
Endometrioid Adenocarcinoma

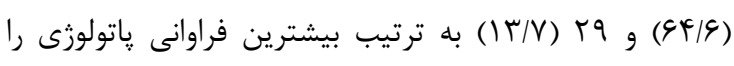

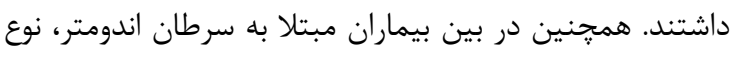
ndometrioid Adenocarcinoma

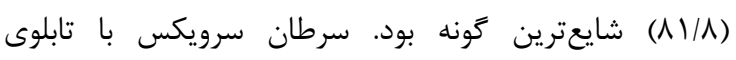

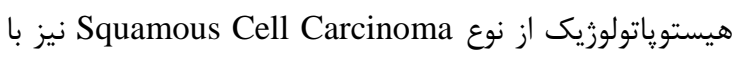

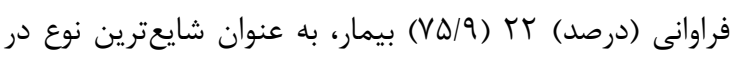

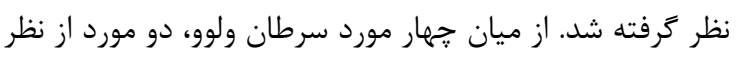

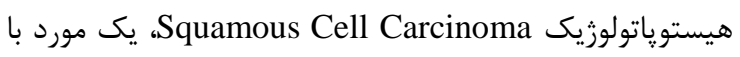
Malignant و و يك مورد با Malignant Melanoma of Vulva Spindle Cell Sarcoma
سرطان دومين علت مرك زنان در سطح جهان مى باشد و به

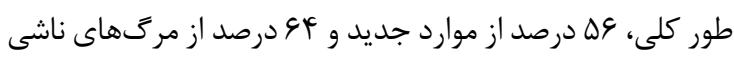

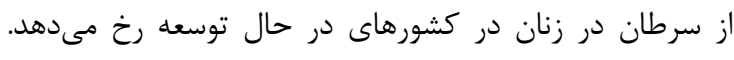

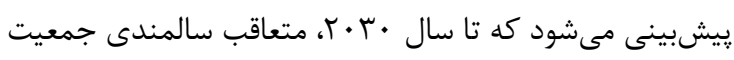

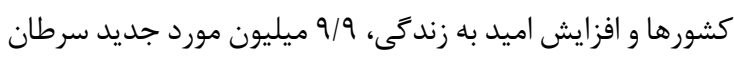

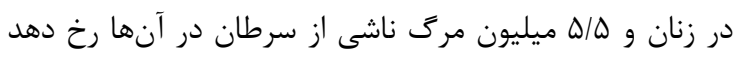

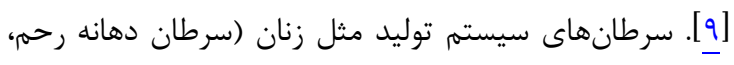

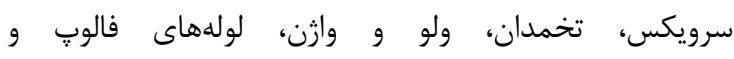

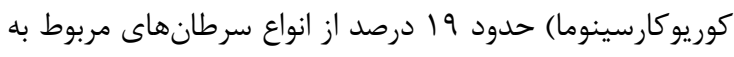

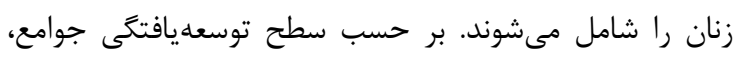

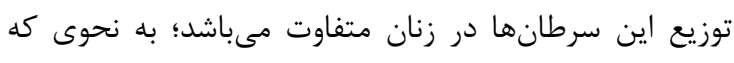

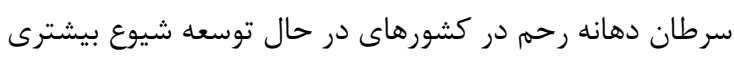

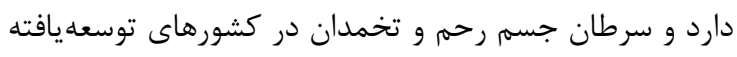

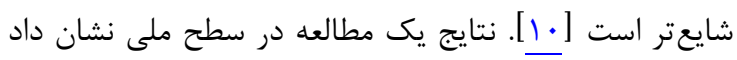

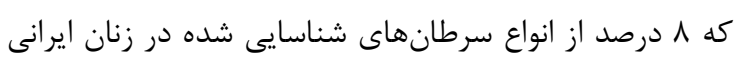

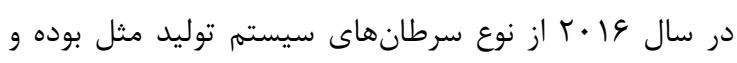

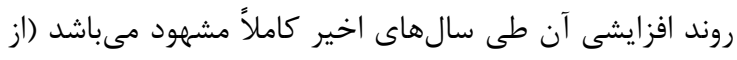

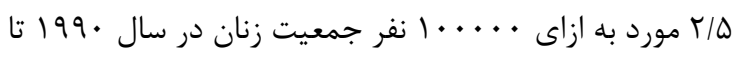

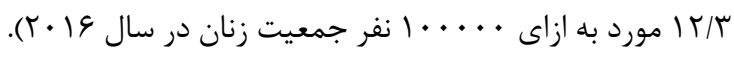

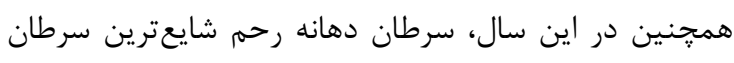

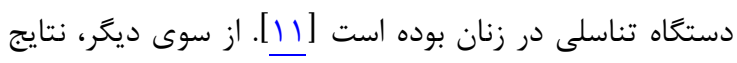

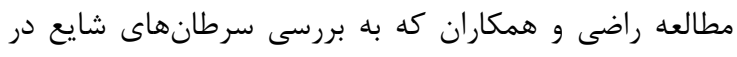

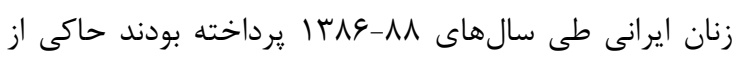

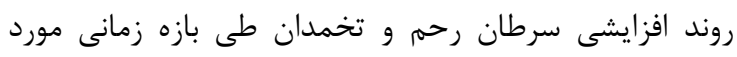

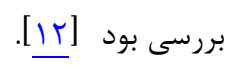

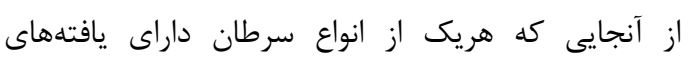

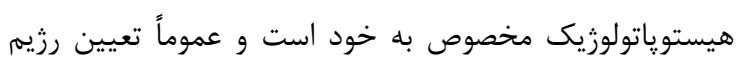

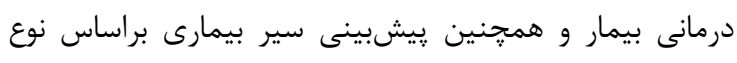

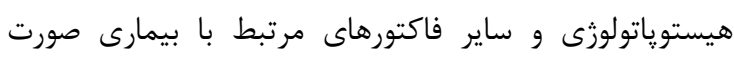

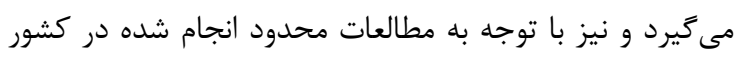

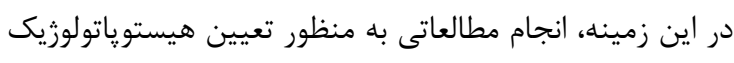

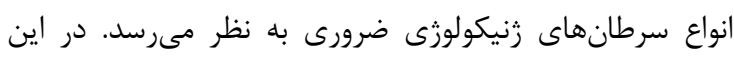

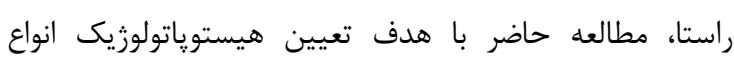

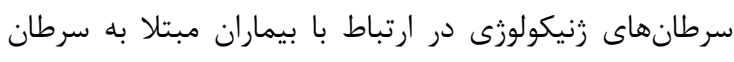

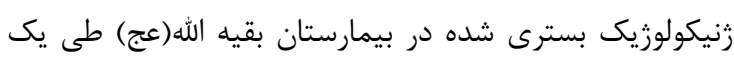
بازه زمانى نه ساله انجام شد.

\section{مواد و روشها}

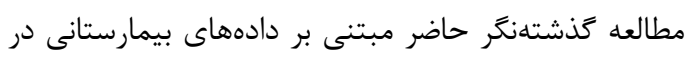

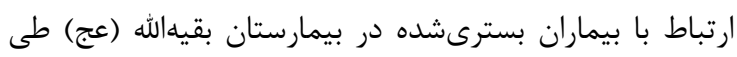

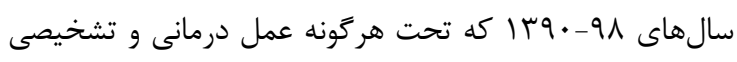

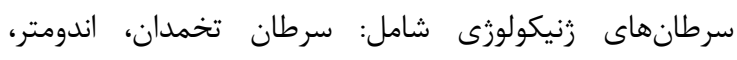

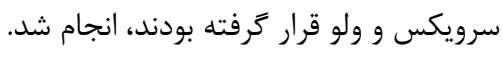

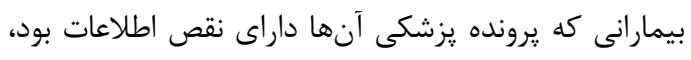


جدول ا: فراوانى انواع هيستوياتولوزى سرطانهاى زنيكولوزيك .

\begin{tabular}{|c|c|c|c|}
\hline تعداد (צ' تحس) & تعداد (وهاد) & تحداد (r/Y) تخدان & انواع هيستوياتولوخى \\
\hline & $9(\Delta / V)$ & $\operatorname{lrV}(\varepsilon F / \varepsilon)$ & Serous Adenocarcinoma \\
\hline$\Delta(I V / T)$ & $\| r \cdot(\lambda) / \Lambda)$ & $r q(I r / V)$ & Endometrioid Adenocarcinoma \\
\hline- & - & 19 (9) & Mucinous Adenocarcinoma \\
\hline- & - & $\varphi(T / \Lambda)$ & Granulosa cell tumor \\
\hline- & $\Delta(r / 1)$ & $\Delta(Y / F)$ & Clear cell Adenocarcinoma \\
\hline- & - & $f(1 / 9)$ & Dysgerminoma \\
\hline- & - & $f(1 / 9)$ & Metastatic Carcinoma \\
\hline$T Y(V Q / Q)$ & $f(Y / \Delta)$ & $r(1 / 4)$ & Squamous Cell Carcinoma \\
\hline- & - & $r(\cdot / 9)$ & Mature teratoma \\
\hline $1(r / \mathbb{F})$ & - & $r(1 / \mathbb{F})$ & Unknown \\
\hline $1(r / \mathbb{Y})$ & - & - & Low grade adenosarcoma \\
\hline- & $r(1 / r)$ & - & Adenosquamous Carcinoma \\
\hline- & $1(\cdot / 9)$ & - & Choriocarcinoma \\
\hline- & $r(1 / 9)$ & - & Mixed Malignant Mullerian Tumor \\
\hline- & $\Delta(r / 1)$ & - & Endometrial Stromal Sarcoma \\
\hline
\end{tabular}

در مقايسه با سرطان سرويكس (اختلاف ميانگين سنى= ع ه/ه،

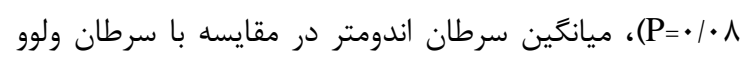

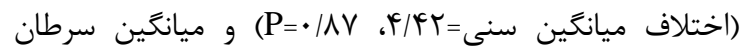
سرويكس در مقايسه با سرطان ولوو (اختلاف ميانغين

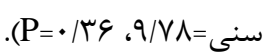

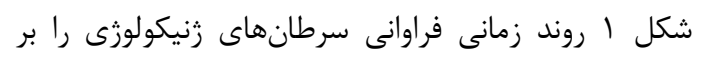

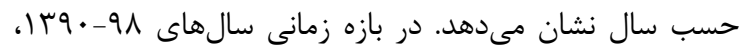

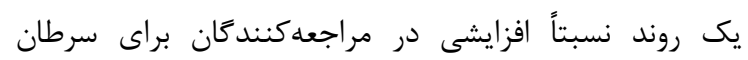
تخمدان اتفاق افتاده بود؛ به طورى كه بيشترين و كمترين دورين موارد

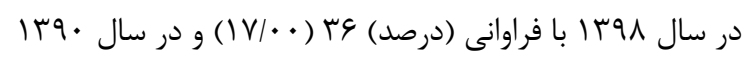

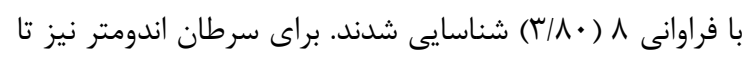

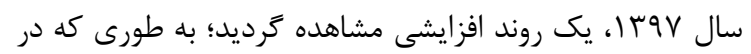

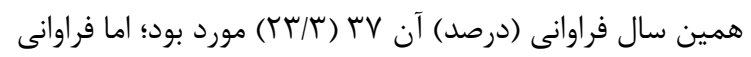

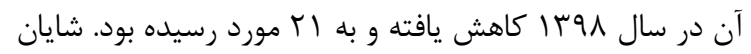

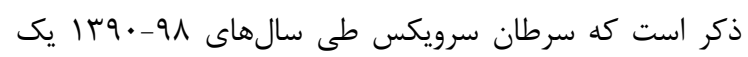

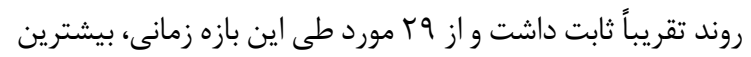

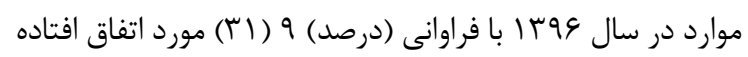

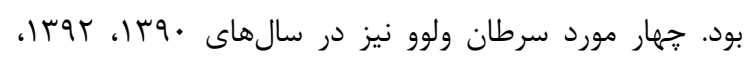

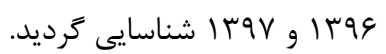

انواع سرطانهاى زنيكولوزيك از نظر هيستولوزيك در جدول 1 ارائه شده است.

در جدول r توزيع سنى بيماران بر حسب سرطان زنيكولوزيك با شاخصهاى مركزى و يراكندگى قابل مشاهده مى باشد. ميانكين

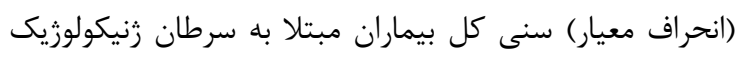

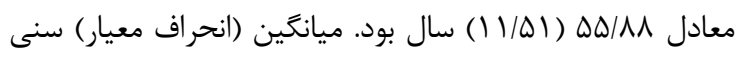

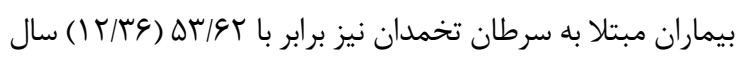

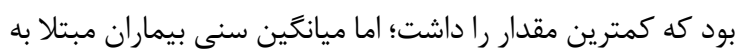

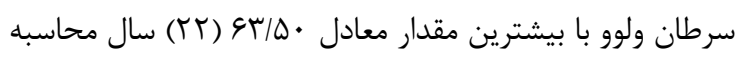

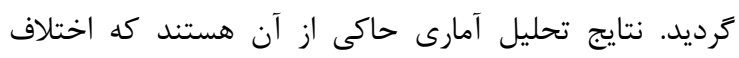

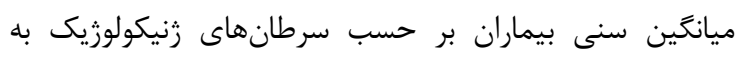

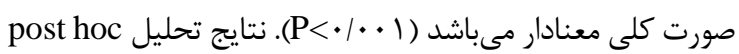
براى مقايسه دوكانه اختلاف ميانكين سنى بيماران مبتلا بـانه

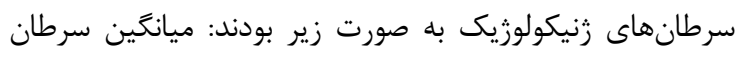

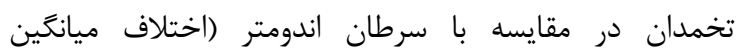

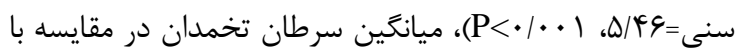

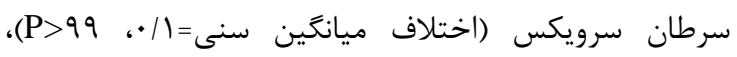
ميانكين سرطان تخمدان در مقايسه با سرطان ولوو (اختلاف

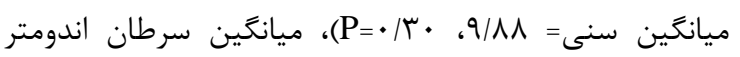

جدول r: توزيع سنى (سال) بيماران بر حسب سرطان زنيكولوزيك

\begin{tabular}{|c|c|c|c|c|c|c|}
\hline سطح معنادارى & انحراف معيار & ميانه & ميانكَين & حداكثر & حداقل & سرطان زنيكولوزى \\
\hline \multirow{4}{*}{$<\cdot / \cdot \cdot 1$} & $\mid r / T 4$ & $\Delta F$ & $\Delta r / G T$ & $\Lambda \Delta$ & IV & تخمدان \\
\hline & $9 / 99$ & $\Delta \Lambda$ & $\Delta q / \cdot \wedge$ & $\Lambda F$ & $r \Lambda$ & اندومتر \\
\hline & N/TY & $\Delta F$ & $\Delta T / V T$ & 99 & rF & سرويكس \\
\hline & $r T / \cdot$ & $99 / 0$. & $q \mu / \Delta$. & $\Lambda F$ & rV & ولوو \\
\hline
\end{tabular}


نسبى سرطانهاى زنيكولوزيك طى سالهاى |q

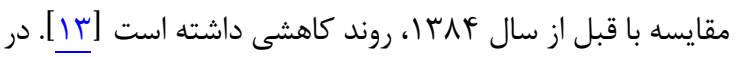

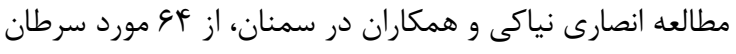

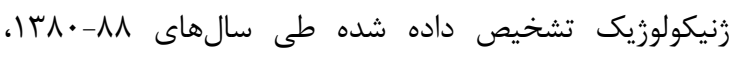

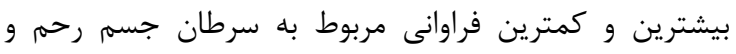

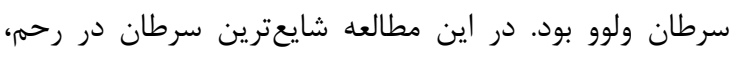

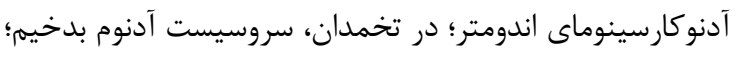

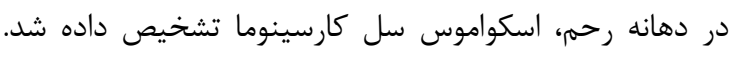

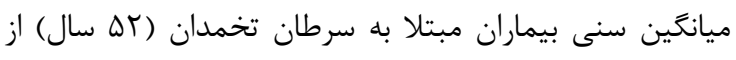

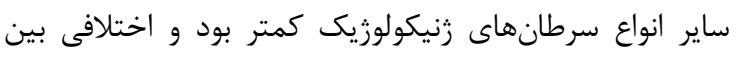

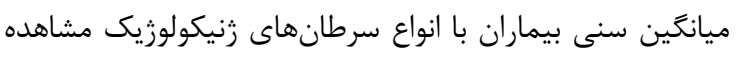

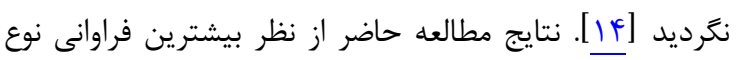

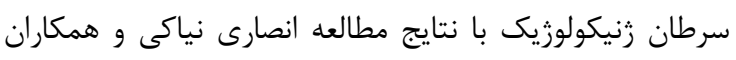

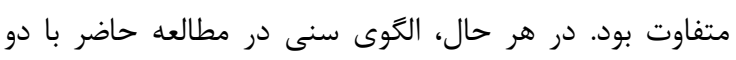

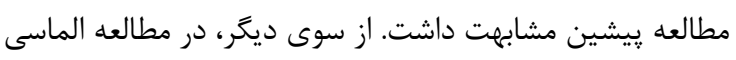

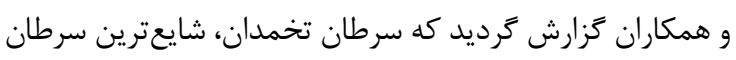

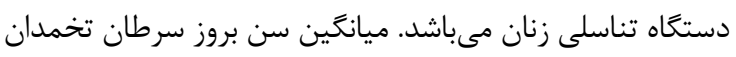

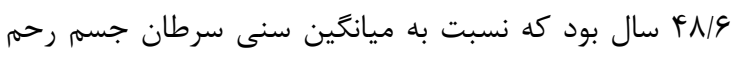

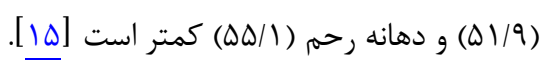

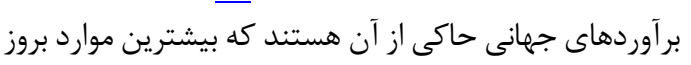

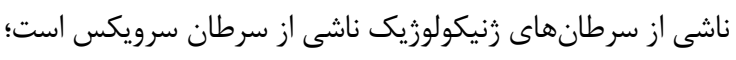

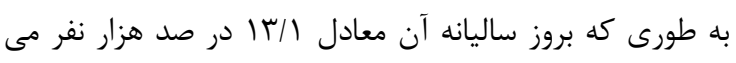

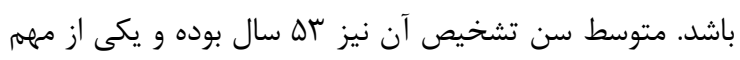

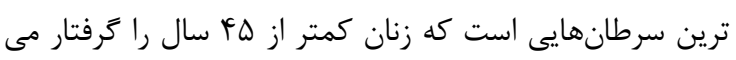

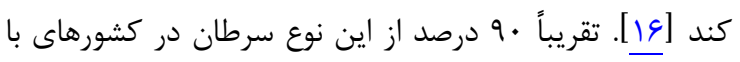

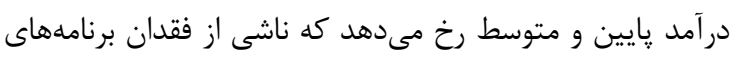

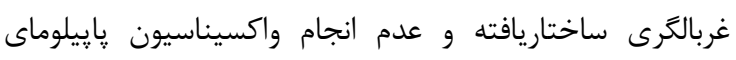

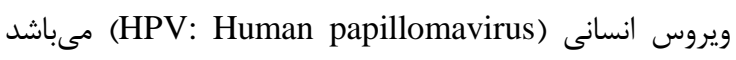

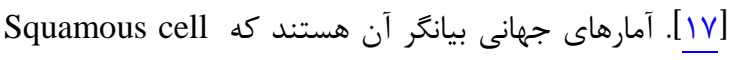
carcinoma

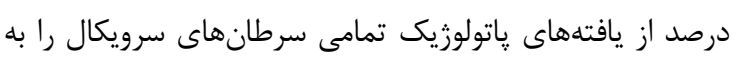

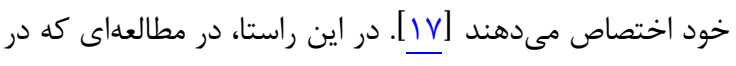

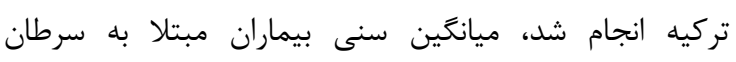

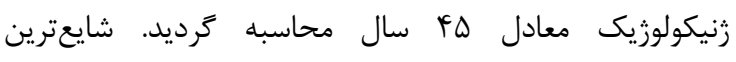

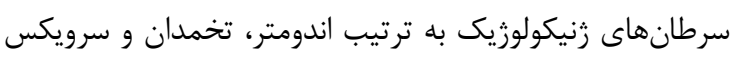

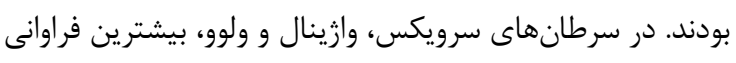

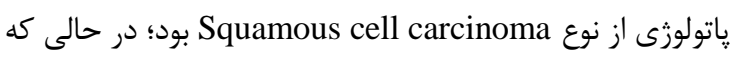
در سرطانهاى تخمدان و اندومتر، بيشترين فراوانى ياتولوزى به داله Endometrioid , Serous adenocarcinoma adenocarcinoma مطالعاتى كه در قاره آفريقا انجام شد، سرطان سرويكس شايعتر ترين نوع سرطان زنيكولوزيك بود و Squamous cell carcinoma

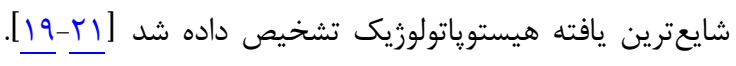

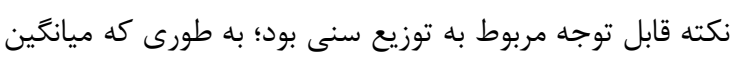

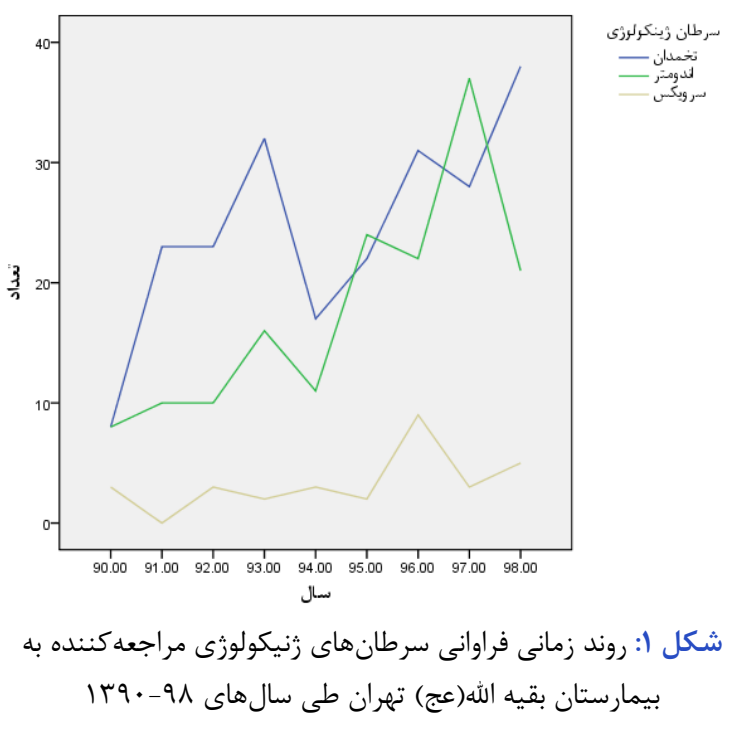

بִ بـ

نتايج مطالعه حاضر نشان دادند كه سرطان تخمدان از نوع Serous Adenocarcinoma

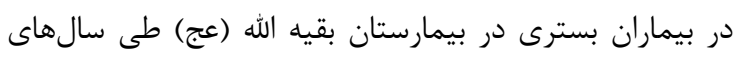

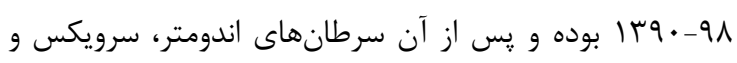

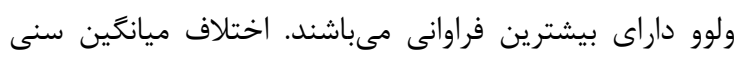

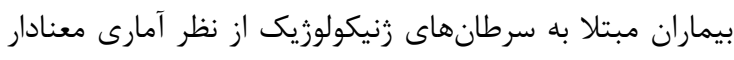

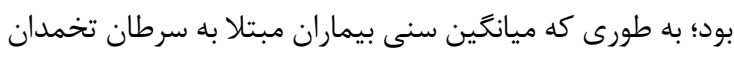

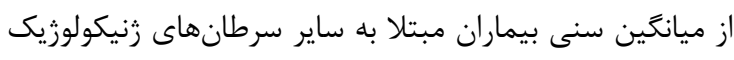

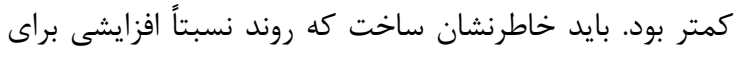

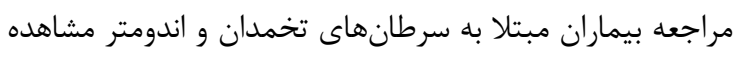

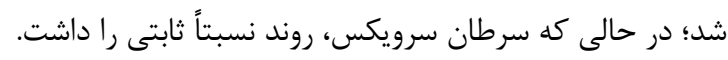

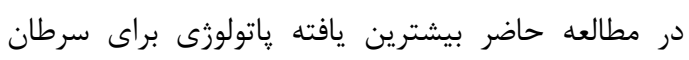

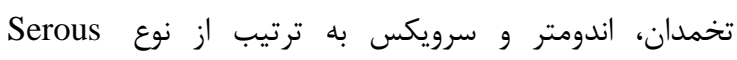
و Endometrioid Adenocarcinoma ،Adenocarcinoma Squamous Cell Carcinoma

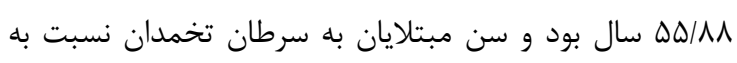

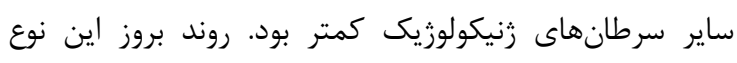

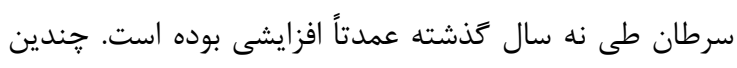

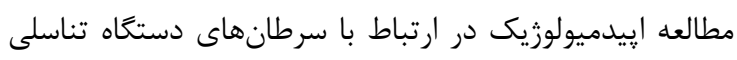

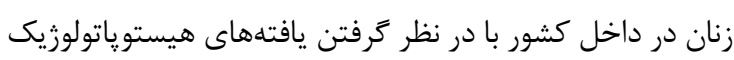

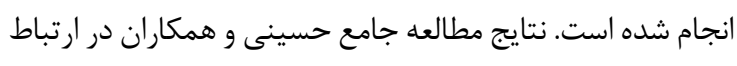

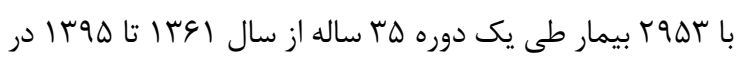

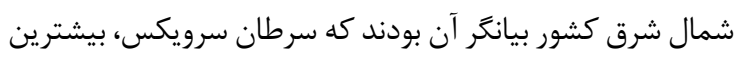

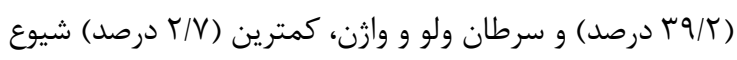

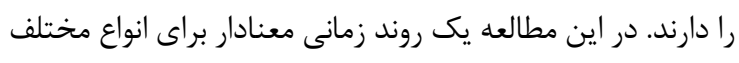

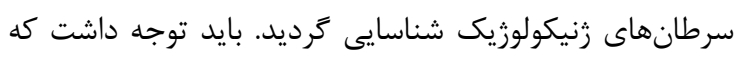

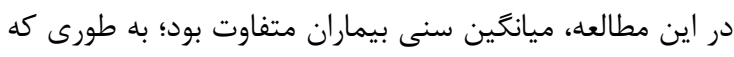

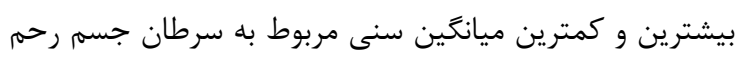

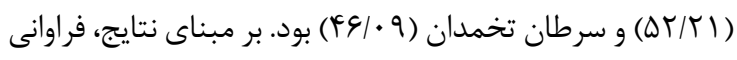


نتيجه تيرى

يافتههاى مطالعه حاضر بيانكَر آن بودند كه سرطان تخمدان

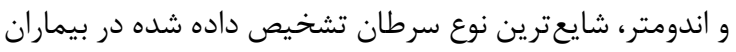

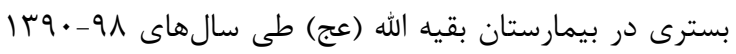

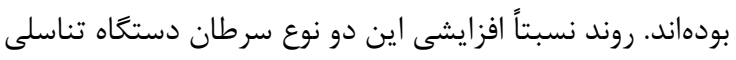

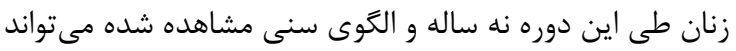

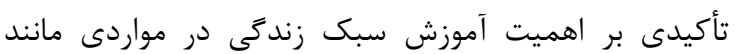

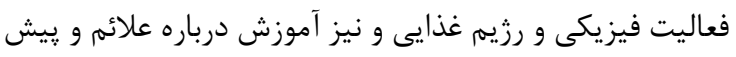

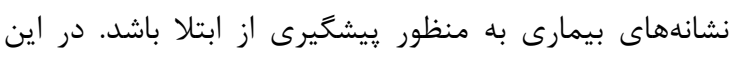

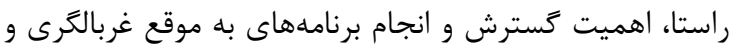

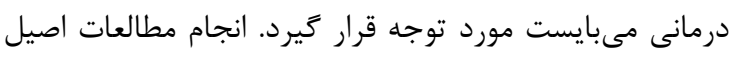
حتى در سطح كشورى در مورد شناخت الكوى إيبدميولوزيك

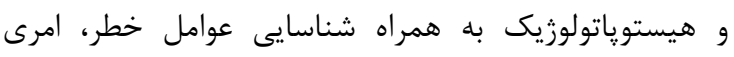

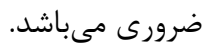

\section{تشكر و قدر مانى}

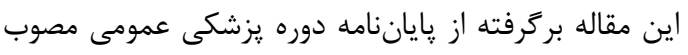

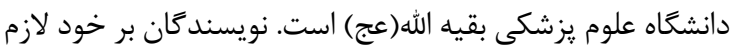

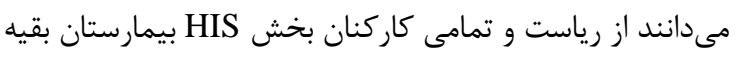
الله(عج) به دليل همكارى در راستاى انجام اين يزوهش، تشارئ تشكر و

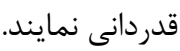

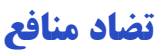
نويسند كان هيجَّونه تضاد منافعى در اين مطالعه ندارند.

ملاحظات اخلاقى اين مطالعه داراى تأييديه از كميته اخلاق دانشكاه علوم

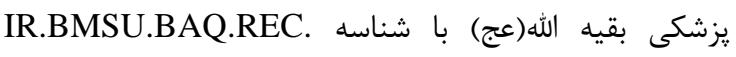
1398.048 مى باشد.

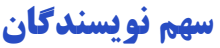

نويسنده اول (يزوهشكر اصلى): طراحى يروزه، تدوين

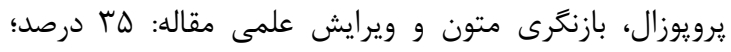

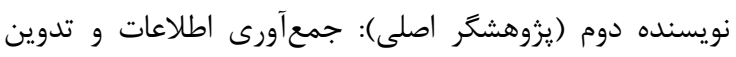

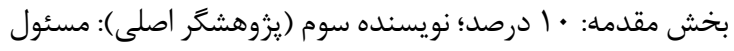

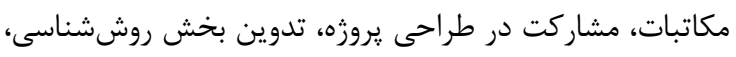

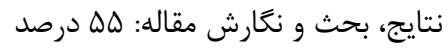

طرح حاضر از سوى دانشكاه علوم يزشكى بقيه الله(عج) تأمين

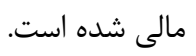

سنى مبتلايان به سرطان ولوو نسبت به ساير انواع سرطانهاى

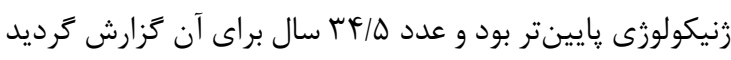

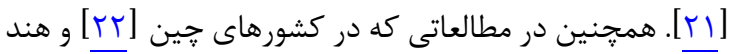

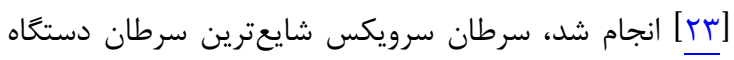

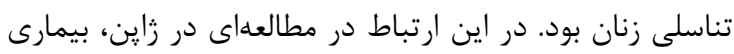

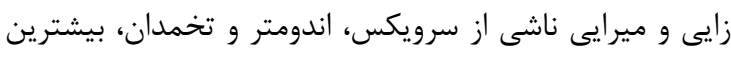

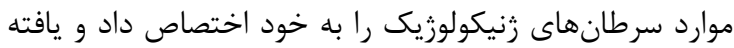

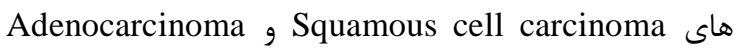

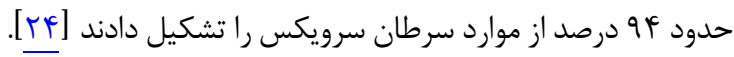

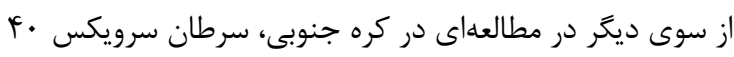

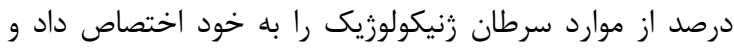

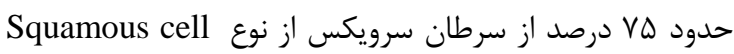
Adenocarcinoma g carcinoma

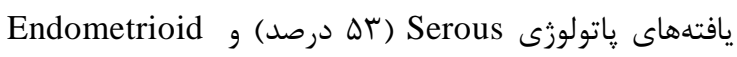
adenocarcinoma

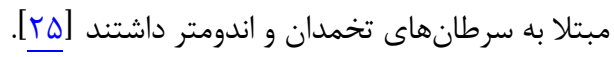

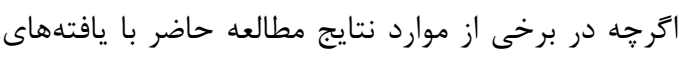

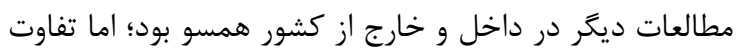

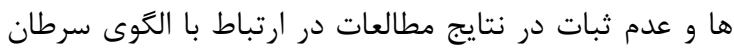

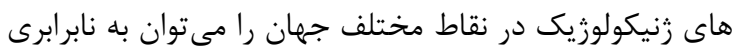

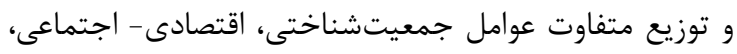

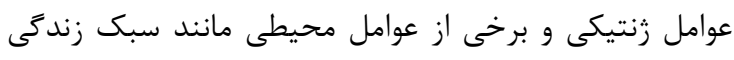

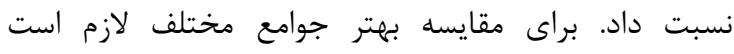

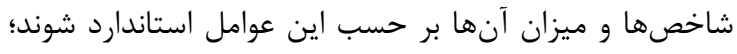

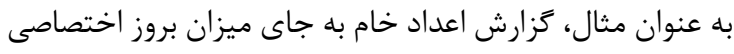

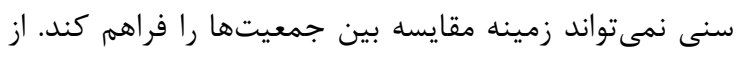

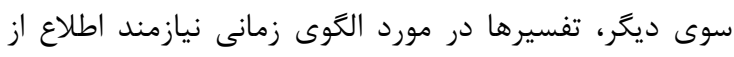

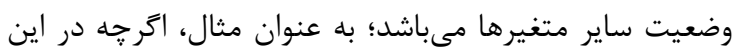

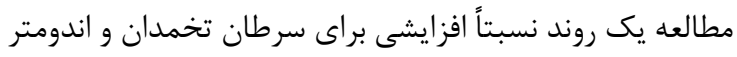

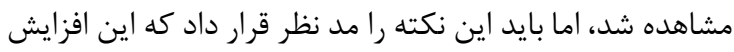

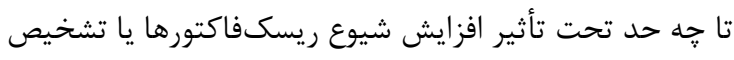
بيشتر بيمارى است. در انتها، بايد خاطرنشان ساخت كه يافتههاى مطالعه حاضر

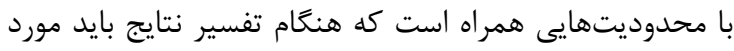

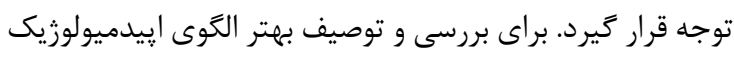

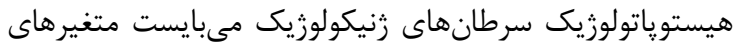

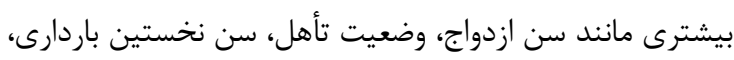

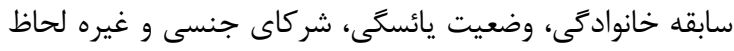

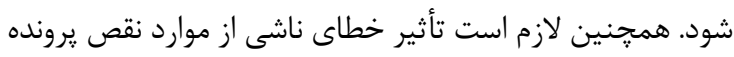

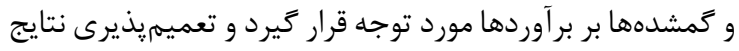

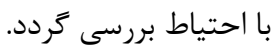

\section{REFERENCES}

1. Goli A, Oroei M, Jalalpour M, Faramarzi H, Askarian M. The spatial distribution of cancer incidence in fars province: a 
2. Merletti F, Galassi C, Spadea T. The socioeconomic determinants of cancer. Environ Health. 2011;10(Suppl 1):S7. PMID: 21489217 DOI: 10.1186/1476-069X-10-S1-S7

3. Sadjadi A, Nouraie M, Mohagheghi M, Mousavi-Jarrahi A, Malekezadeh R, Parkin D. Cancer occurrence in Iran in 2002, an international perspective. Asian Pac J Cancer Prev. 2005;6:359-63. PMID: 16236000

4. Babaei M, Mousavi S, Malek M, Tosi G, Masoumeh Z, Danaei N, et al. Cancer occurrence in Semnan Province, Iran: Results of a population-based cancer registry. Asian Pac J Cancer Prev. 2005;6(2):159-64. PMID: 16101326

5. Cramb S, Mengersen K, Baade P. Developing the atlas of cancer in Queensland: methodological issues. Int J Health Geogr. 2011;10:9. PMID: 21261992 DOI: 10.1186/1476072X-10-9

6. Mousavi SM, Gouya MM, Ramazani R, Davanlou M, Hajsadeghi N, Seddighi Z. Cancer incidence and mortality in Iran. Ann Oncol. 2011;20(3):556-63. DOI: 10.1093/ annonc/mdn642

7. Masoompour S, Yarmohammadi $\mathrm{H}$, Rezaianzadeh $\mathrm{A}$, Lankarani K. Cancer incidence in southern Iran, 1998-2002: Results of population-based cancer registry. Cancer Epidemiol. 2011;35(5):e42-7. PMID: 21840285 DOI: 10.1016/j.canep.2011.05.018

8. Mehrabani D, Tabei SZ, Heydari ST, Shamsina SJ, Shokrpour N, Amini M, et al. Cancer occurrence in Fars Province, Southern Iran. Iran Red Crescent Med J. 2008; 10(4):314-22.

9. Torre LA, Islami F, Siegel RL, Ward EM, Jemal A. Global cancer in women: burden and trends. Cancer Epidemiol Biomarkers Prev. 2017;26(4):444-57. PMID: 28223433 DOI: 10.1158/1055-9965.EPI-16-0858

10. Sankaranarayanan R, Ferlay J. Worldwide burden of gynaecological cancer: the size of the problem. Best Pract Res Clin Obstet Gynaecol. 2006;20(2):207-25. PMID: 16359925 DOI: 10.1016/j.bpobgyn.2005.10.007

11. Rajai N, Ghanbari A, Yoosefi M, Mohebi F, Mohajer B, Sheidaei A, et al. National and subnational trends in incidence and mortality of lung cancer in Iran from 1990 to 2016. Asia Pac J Clin Oncol. 2020;16(3):129-36. PMID: 32030897 DOI: $10.1111 /$ ajco. 13303

12. Razi S, Salehiniya H, Fathali Loy Dizaji M. Epidemiology of prevalent cancer among Iranian women and its incidence trends from 2003-2009 in Iran. J Arak Univ Med Sci. 2015; 18(2):17-24

13. Hoseini SS, Yousefi Z, Emadzadeh M, Homaee Shandiz F, Hokmabadi P, Salehi M, et al. Changes in prevalence and epidemiology of female genital cancers in northeastern Iran in a 35-year study. Iran J Obstet Gynecol Infertil.
2020;22(12):11-8.

14. Ansariniaki M, Ghorbani R, Semnani V, Pahlavan S. Epidemiological-histopathological status of gynecological cancers in Iranian population: a 9-year study. Koomesh. 2017;19(2):484-91.

15. Almasi-Hashiani A, Farahmand M. Trend of incidence rate for female genital cancers based on cancer registry data in Fars province during 2003-2009. J Feyz. 2012;16(4):353-60.

16. Arbyn M, Weiderpass E, Bruni L, de Sanjosé S, Saraiya M, Ferlay $\mathbf{J}$ et al. Estimates of incidence and mortality of cervical cancer in 2018: a worldwide analysis. Lancet Global Health. 2020;8(2):e191-203. PMID: 31812369 DOI: 10.1016/S2214109X(19)30482-6

17. Cohen PA, Jhingran A, Oaknin A, Denny L. Cervical cancer. Lancet. 2019;393(10167):169-82. PMID: 30638582 DOI: 10.1016/S0140-6736(18)32470-X

18. Dhakal HP, Pradhan M. Histological pattern of gynecological cancers. JNMA J Nepal Med Assoc. 2009;48(176):301-5. PMID: 21105554

19. Hailu HE, Mondul AM, Rozek LS, Geleta T. Descriptive epidemiology of breast and gynecological cancers among patients attending Saint Paul's Hospital Millennium Medical College, Ethiopia. PloS One. 2020;15(3):e0230625. PMID: 32196536 DOI: 10.1371/journal.pone.0230625

20. Ibrahim H, Ijaiya MA. Pattern of gynaecological malignancies at the University of Ilorin Teaching Hospital, Ilorin, Nigeria. J Obstet Gynaecol. 2013;33(2):194-6. PMID: 23445148 DOI: 10.3109/01443615.2012.738717

21. Osinachi I, Adewole N, Isah A, Abdullahi H, Agida E. Pattern of gynaecological malignancies in a Nigerian tertiary hospital. Afr J Med Health Sci. 2020;19(3):29-35

22. Jiang $X$, Tang H, Chen T. Epidemiology of gynecologic cancers in China. J Gynecol Oncol. 2018;29(1):e7. PMID: 29185265 DOI: 10.3802/jgo.2018.29.e7

23. Barman D, Sharma JD, Barmon D, Kataki AC, Sharma A, Kalita M. Epidemiology of gynecological cancers in Kamrup Urban District cancer registry. Indian $J$ Cancer. 2017;54(1):388-91. PMID: 29199729 DOI: 10.4103/0019509X.219590

24. Yamagami W, Nagase S, Takahashi F, Ino K, Hachisuga T, Aoki D et al. Clinical statistics of gynecologic cancers in Japan. J Gynecol Oncol. 2017;28(2):e32. PMID: 28198168 DOI: 10.3802/jgo.2017.28.e32

25. Lee SY, Kim E, Kim HS, Koo YJ, Lee DH. Clinical and histopathologic analysis of gynecological cancer: a single institute experience over 7 years. Yeungnam Univ J Med. 2020;37(3):179-85. PMID: 32131080 DOI: 10.12701/ yujm.2019.00451 